\title{
Die Impfung hat die beste Wirkung im Ziel
}

\section{Urs Stoffel}

Dr. med., Mitglied des FMH-Zentralvorstandes, Departementsverantwortlicher Ambulante Versorgung und Tarife

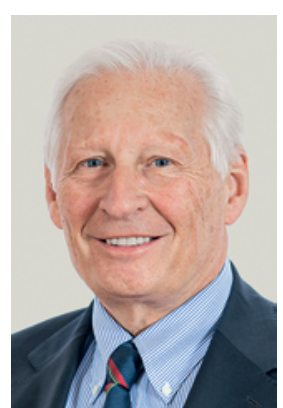

Seit Wochen beherrscht das Dauerthema Covid-19Impfungen die Schlagzeilen und ist Tagesgespräch. Anfang des Jahres wurden die Kantone von der raschen Verfügbarkeit des Impfstoffes überrascht und waren nicht vollständig bereit für die Umsetzung der geplanten Impfkampagne. Als man dann so weit war, stockte prompt der Nachschub an Impfstoff. Damit konnte die Impfstrategie bisher nicht so zügig wie gewünscht umgesetzt werden. Auch die Entschädigung für die Impfungen in den Impfzentren und Arztpraxen hätte besser vorbereitet werden können.

Die Arztpraxen waren primär gar nicht oder erst viel später für die Impfung der Bevölkerung vorgesehen. Jedoch zeigte sich bereits in der ersten Phase der Umsetzung, in der die älteren, chronisch kranken und

\section{Gerade Risikopatienten möchten sich gerne} in der Praxis von ihrer wohlbekannten behandelnden Ärztin impfen lassen.

polymorbiden Patienten geimpft werden sollten, dass der Ärzteschaft in der freien Praxis eine wichtige Rolle zukommt. Denn es sind die Ärztinnen und Ärzte in den Praxen, welche die Priorisierung und die Triage wie auch die Aufklärung dieser Hochrisikopatienten übernehmen. Sei es, um ihre Patientinnen und Patienten gezielt in ein Impfzentrum zu überweisen, sei es, um sie selbst in ihrer Arztpraxis zu impfen. Das Bedürfnis, sich in der gewohnten Arztpraxis impfen zu lassen, ist gerade bei diesen Hochrisikopatienten gross. Sie wollen bei ihrer wohlbekannten betreuenden Ärztin oder ihrem betreuenden Arzt geimpft werden und nicht in einem Impfzentrum. Es ist eine Frage des Vertrauens. Risikopatienten haben Fragen und wollen beraten werden. Sie sind die vulnerabelste Bevölkerungsgruppe in dieser Pandemie.

Nicht nachvollziehbar ist das Vorgehen der Versicherer und der Gesundheitsdirektorenkonferenz (GDK), wenn es um die Impfentschädigung geht. Anfang Januar haben die Vertragspartner GDK und die Versicherer einen Tarifvertrag abgeschlossen. Darin wurde auch die Höhe der Impfpauschale von CHF 14.50 geregelt, die sowohl für die Impfzentren als auch für Arztpraxen gültig sind. Die FMH war aber zu keiner Zeit Vertragspartner und konnte auch keine eigentlichen Preisverhandlungen führen, obwohl im Vertrag auch die Entschädigung für Impfungen in den Arztpraxen geregelt wird. Das ist ein klassischer Vertrag zu Lasten Dritter. Die FMH hat sich zusammen mit dem Verband der Haus- und Kinderärzte mfe nach Bekanntgabe der Entschädigung eingeschaltet. Wir wurden angehört und konnten mit klaren Berechnungen aufzeigen, dass eine Impfpauschale von CHF 14.50 in den Arztpraxen niemals kostendeckend sein kann. Auch in den Impfzentren werden die Kosten ein Mehrfaches dieser CHF 14.50 betragen; entsprechend wird die Impfung über Steuergelder quersubventioniert. Hinter verschlossenen Türen haben die Versicherer und die GDK daraufhin eine vorläufige Impfpauschale von CHF 24.50 für Arztpraxen ausgehandelt. Damit wurde das Ziel einer einheitlichen und kostendeckenden Impfpauschale für alle Arztpraxen in der Schweiz bei weitem verfehlt. Somit werden sich Impfpauschalen für Arztpraxen von Kanton zu Kanton unterscheiden, da die Kantone, verantwortlich für der Umsetzung der Impfkampagne, nun die Impfpauschalen für eine angemessene Versorgung ihrer Bürger nachbessern müssen. Die kantonalen Ärztegesellschaften müssen jeweils einzeln mit den Kantonen verhandeln. Genau das wollten wir eigentlich vermeiden.

Mit der Covid-Impfung verfügen wir erstmals über ein Instrument, das uns erlaubt, mit der grössten Wirkung im Ziel aus dieser Pandemie herauszukommen. Unverständlich und geradezu grotesk ist, dass wir ausgerechnet jetzt um knapp kostendeckende Impfpauschalen in den Arztpraxen kämpfen müssen. Täglich werden rund 100 Millionen vom Staat ausgegeben, um die wirtschaftlichen Auswirkungen der Pandemiemassnahmen zu lindern. Diese Massnahmen könnten gelockert werden, wenn wir mit der Impfung rasch voranschreiten, da die Impfung einen wirksamen Schutz zur Lösung des Problems darstellt. Erfreulich ist, dass wenigstens ein Teil der Kantone, im Gegensatz zu den Versicherern, ihre Verantwortung wahrnehmen und inzwischen eine honorierende Pauschale für die Arztpraxen beschlossen haben. 